Pesq. Vet. Bras. 29(3):233-240, março 2009

\title{
Intoxicação espontânea por vagens de Prosopis juliflora (Leg. Mimosoideae) em bovinos no Estado de Pernambuco ${ }^{1}$
}

\author{
Antônio Carlos L. Câmara ${ }^{2 *}$, Nivaldo de Azevêdo Costa ${ }^{3}$, Franklin Riet- \\ Correa ${ }^{4}$, José Augusto B. Afonso ${ }^{3}$, Antônio F. M. Dantas ${ }^{4}$, Carla Lopes de \\ Mendonça ${ }^{3}$ e Maria Isabel de Souza ${ }^{3}$
}

\begin{abstract}
Câmara A.C.L., Costa N.A., Riet-Correa F., Afonso J.A.B., Dantas A.F.M., Mendonça C.L. \& Souza M.I. 2009. [Spontaneous poisoning in cattle by mesquite beans, Prosopis juliflora (Leg. Mimosoideae) in Pernambuco.] Intoxicação espontânea por vagens de Prosopis juliflora (Leg. Mimosoideae) em bovinos em Pernambuco. Pesquisa Veterinária Brasileira 29(3):233-240. Clínica de Bovinos, Campus Garanhuns, Universidade Federal Rural de Pernambuco, Av. Bom Pastor s/n, Cx. Postal 152, Mundaú, Garanhuns, PE 55292-901, Brazil. E-mail: aclcamara@yahoo.com.br

Three outbreaks of poisoning by Prosopis juliflora pods are reported in the semiarid region of the state of Pernambuco, Northeastern Brazil, in cattle grazing in fields invaded by the plant or ingesting mesquite beans as a concentrate food. In two farms the disease occurred sporadically. In another, 112 (9.28\%) cattle out of 1206 were affected, 84 (6.96\%) died due to emaciation, and $28(2.32 \%)$ gained weight after the pods had been withdrawn from the feed. Main clinical signs were progressive weight loss, atrophy of the masseter muscles, dropped jaw, tongue protrusion, difficulties in prehending food, tilting the head during mastigation or rumination, salivation, impaired swallowing, and decreased tone of the tongue. The hematology reveals hypoproteinemia and anemia. Gross lesions were emaciation and reduction in size of the masseter muscles, which appear thinner than normal and grayish due muscular atrophy. Degeneration of neurons of the trigeminal motor nuclei, Wallerian degeneration of the trigeminal nerve roots, and muscular atrophy of the masseter muscles with substitution by fibrous tissue were observed on histologic examination. For the prevention of the poisoning is necessary to limit the amount of mesquite beans in animal nutrition. It is also necessary to develop research to determine the economic and sustainability of the use of Prosopis juliflora for animal food, human food or other uses such as charcoal, wood and fuel wood.
\end{abstract}

INDEX TERMS: Poisonous plants, Prosopis juliflora, Leguminosae Mimosoideae, plant poisoning, cattle, cranial nerves impairment, neuronal degeneration.

RESUMO.- Descrevem-se três surtos de intoxicação por vagens de Prosopis juliflora no Sertão e Agreste de Pernambuco, na região semi-árida, em animais pastejan-

\footnotetext{
${ }^{1}$ Recebido em 3 de novembro de 2008.

Aceito para publicação em 1 de dezembro de 2008.

2 Programa de Pós-Graduação em Saúde Animal, Faculdade de Agronomia e Medicina Veterinária, Universidade de Brasília, Galpão 4, Granja do Torto, Brasília, DF 70636-200, Brasil. *Autor para correspondência: aclcamara@yahoo.com.br

${ }^{3}$ Clínica de Bovinos, Campus Garanhuns, Universidade Federal Rural de Pernambuco, Cx. Postal 152, Garanhuns, PE 55292-901, Brasil.

${ }^{4}$ Hospital Veterinário, Centro de Saúde e Tecnologia Rural, Campus de Patos da Universidade Federal de Campina Grande, Patos, PB 58700-000, Brasil.
}

do áreas invadidas pela planta ou que ingeriram as vagens como alimento concentrado. Em duas fazendas nas que a doença ocorria esporadicamente foram observados casos individuais. Em outra, o surto afetou um rebanho de 1206 bovinos, dos quais adoeceram $112(9,28 \%)$ e morreram $84(6,96 \%)$, enquanto os demais $28(2,32 \%)$ recuperaram-se e ganharam peso após a retirada das vagens da alimentação. Clinicamente observou-se, principalmente, perda de peso progressiva, atrofia da musculatura da face e masseter, mandíbula pendulosa, protrusão de língua, dificuldade de apreensão e mastigação dos alimentos, torção da cabeça para mastigar ou ruminar, salivação excessiva, disfagia e hipotonia lingual. Nos exames laboratoriais constatou-se anemia e hipoproteinemia. 
$\mathrm{Na}$ necropsia havia caquexia e diminuição de volume e coloração acinzentada dos músculos masseteres. $\mathrm{Na}$ histologia observou-se degeneração de neurônios do núcleo motor do trigêmeo, degeneração Walleriana do nervo trigêmeo e atrofia muscular por denervação do músculo masseter com substituição por tecido fibroso. Recomendam-se medidas para a profilaxia da intoxicação e discute-se a necessidade de desenvolver pesquisas para determinar a viabilidade econômica e sustentabilidade da utilização da algaroba como alimento animal ou humano e para produção de carvão, lenha ou madeira.

TERMOS DE INDEXAÇÃO: Plantas tóxicas, Prosopis juliflora, Leguminosae Mimosoideae, intoxicação por planta, bovinos, insuficiência de nervos cranianos, degeneração neuronal.

\section{INTRODUÇÃO}

Prosopis juliflora (algaroba ou algarobeira) foi introduzida no Brasil no Estado de Pernambuco, na década de 1940, a partir de sementes oriundas do Peru (Azevedo 1961). Desde então, devido à combinação de baixos custos, alta palatabilidade e valores nutricionais, principalmente das vagens, estas vêm sendo usadas como suplemento ou parte integrante de rações na alimentação de diversas espécies de animais domésticos, dentre elas, codornas, galinhas poedeiras, eqüinos, suínos, peixes e ruminantes. Além disso, também tem sido utilizada para o consumo humano, como pães, biscoitos e doces (Ravikala et al. 1995, Silva et al. 2002a,b, Tabosa et al. 2004, Mahgoub et al. 2005a,b, Stein et al. 2005).

A ingestão das vagens da algarobeira tem sido reconhecida, no Nordeste do Brasil, como causa de uma doença de bovinos que tem o nome popular de "cara torta" devido ao desvio lateral de cabeça que o animal realiza para manter o alimento na boca durante a mastigação. Anteriormente, porém, já se havia comprovado que a ingestão das vagens dessa planta era responsável pela ocorrência de uma enfermidade denominada "jaw and tongue trouble" em bovinos nos Estados Unidos (Dollahite 1964, Kingsbury 1964) e "coquera" em caprinos no Peru (Bacca et al. 1963/66). A intoxicação ocorre, também, com o denominado farelo de algaroba, que se obtém por moagem das vagens após secagem adequada (Tabosa et al. 2006). No Brasil, a doença foi descrita em bovinos no Rio Grande do Norte (Silva et al. 2006), Paraíba e Pernambuco (Dantas \& Menezes 1994), e em caprinos na Paraíba (Lima et al. 2004).

Os sinais clínicos, mais evidentes durante a ruminação, são característicos de disfunção de nervos cranianos, principalmente devido à degeneração e desaparecimento dos neurônios do núcleo motor do trigêmeo (Tabosa et al. 2000a, 2006). Observa-se relaxamento da mandíbula, desvio lateral da cabeça durante a mastigação e ruminação, incoordenação dos movimentos mastigatórios, dificuldade de apreensão dos alimentos, salivação profusa, disfagia e atrofia dos masseteres. Além disso, atonia ruminal, anemia, edema submandibular, e emagrecimento progressivo são, também, observados (Kingsbury 1964,
Figueiredo et al. 1996, Riet-Correa et al. 2002, 2003, Tabosa et al. 2004, 2006). Os caprinos apresentam aumento do tempo de ruminação, salivação, emagrecimento e tremores dos lábios, da mandíbula e da cabeça, principalmente durante a mastigação, o que evidencia debilidade muscular dos músculos da mastigação (Tabosa et al. 2000a, Lima et al. 2004).

Os achados de necropsia são atrofia dos músculos mastigatórios, o que proporciona um aspecto pálido e firme ao tecido muscular, além de diversos graus de desnutrição. Histologicamente, além da atrofia muscular, observa-se degeneração dos neurônios de alguns núcleos do tronco encefálico (núcleo motor do trigêmeo e dos núcleos facial, hipoglosso e oculomotor). Essa degeneração caracteriza-se por fina vacuolização do pericário dos neurônios, que assumem aspecto espumoso com dissolução da substância de Nissl e degeneração axonal (Riet-Correa et al. 2003, Tabosa et al. 2006). Na microscopia eletrônica, os neurônios dos núcleos do trigêmeo apresentam marcante aumento de volume das mitocôndrias, com a crista mitocondrial deslocada perifericamente e desintegrada (Tabosa et al. 2006). As lesões histológicas dos músculos caracterizam-se por atrofia de denervação e substituição das fibras musculares por tecido conjuntivo e tecido adiposo (Riet-Correa et al. 2003, Tabosa et al. 2006). Um trabalho experimental em caprinos demonstrou severa vacuolização de neurônios no núcleo motor do nervo trigêmeo e no núcleo do oculomotor. Adicionalmente havia degeneração Walleriana do nervo trigêmeo e atrofia por denervação dos músculos da mastigação (Tabosa et al. 2000a). Lima et al. (2004) observaram também vacuolização de neurônios motores da medula e do gânglio trigêmeo, dos núcleos motores do trigêmeo, facial e hipogloso. Vacuolização de neurônios motores do núcleo do trigêmeo também foi observada em um caprino que ingeriu folhas, caules e vagens de Prosopis glandulosa (Washburn et al. 2002).

As vagens de Prosopis juliflora contêm alcalóides piperidínicos, mas é incerto se essas substâncias são responsáveis pela vacuolização neuronal, que é a lesão primária da intoxicação (Tabosa et al. 2000b). A intoxicação foi reproduzida experimentalmente em bovinos mediante 0 fornecimento, durante seis meses, de rações que continham $50 \%$ e $100 \%$ de vagens de algaroba (Figueiredo et al. 1996), enquanto Tabosa et al. (2006) obtiveram a intoxicação após ingestão de dietas com 50\% e 75\% das vagens durante 45-75 dias. Os caprinos são mais resistentes e têm que ingerir concentrações de $60-90 \%$ de frutos na alimentação por um período de aproximadamente 210 dias para apresentar sinais clínicos (Tabosa et al. 2000a), já ovinos não se intoxicaram após receber vagens de algaroba a $70-100 \%$ da alimentação por um ano (Lima et al. 2004).

O objetivo deste trabalho é relatar a ocorrência e descrever os achados clínicos e laboratoriais de surtos de intoxicação por vagens de Prosopis juliflora diagnosticados na Clínica de Bovinos, Campus Garanhuns da Universidade Federal Rural de Pernambuco. 


\section{MATERIAL E MÉTODOS}

As informações foram obtidas a partir das fichas de acompanhamento clínico de seis bovinos no período entre outubro de 2006 a agosto de 2007. Foram resgatadas as informações relacionadas aos dados epidemiológicos, obtido junto aos proprietários, além dos achados clínicos e laboratoriais.

Sobre o surto de intoxicação por Prosopis juliflora (fam. Leg. Mimosoideae) em 2007, no município de Custódia-PE, após exame clínico de um dos animais acometidos (Bovino 2) que foi trazido à Clínica de Bovinos de Garanhuns, Universidade Federal Rural de Pernambuco (CBG-UFRPE), os dados referentes aos sinais clínicos e evolução da doença foram obtidos durante visita realizada na propriedade por dois dos autores (ACLC e NAC) na ocasião do surto e, recentemente, foram obtidas informações complementares com o proprietário.

Os três animais atendidos na CBG-UFRPE (Bovinos 1, 2 e 6), assim como os três animais mais severamente acometidos no surto em Custódia-PE (Bovinos 3, 4 e 5) foram examinados clinicamente seguindo as recomendações de Dirksen et al. (1993), enquanto o exame neurológico foi realizado de acordo com Mayhew (1989). Foram coletadas amostras sangüíneas de quatro bovinos, em tubo a vácuo com anticoagulante EDTA (10\%), para realização do hemograma, determinação da proteína plasmática total e do fibrinogênio plasmático, conforme Jain (1993). Devido ao precário estado corpóreo de um dos animais (Bovino 2) que permanecia em decúbito lateral e evoluiu para o quadro pré-comatoso com dispnéia respiratória mista, optou-se pela eutanásia e necropsia. O sistema nervoso central, hipófise, rete mirabile carotídea, músculo masseter, baço, fígado, rim e coração, foram fixados em formalina a $10 \%$, e posteriormente, processados rotineiramente para histologia e corados pela técnica de hematoxilina-eosina $(\mathrm{HE})$. O estudo histológico do sistema nervoso central foi realizado em cortes transversais do córtex cerebral, núcleos da base, cerebelo, tronco encefálico à altura do núcleo do trigêmeo, ponte, óbex e medula cervical. A análise histológica foi realizada no setor de Anatomia Patológica da Universidade Federal de Campina Grande, Campus Patos, Paraíba.

Um estudo caso-controle foi realizado no rebanho da propriedade do município de Custódia-PE, avaliando-se o fator de risco relacionado, no caso raça (Nelore ou mestiço), calculando-se a razão de chances (odds ratio) com um intervalo de confiança de $95 \%$, utilizando-se o programa estatístico Winepiscope 2.0® (Winespiscope 2.0... 2000).

\section{RESULTADOS}

Os dados epidemiológicos dos casos diagnosticados na CBG-UFRPE estão resumidos no Quadro 1. Todos os bovinos eram criados em sistema extensivo, com livre acesso a alimentos volumosos (capim Buffel, capim elefante, grama estrela e/ou pastagem nativa) e amplas áreas com intensa densidade de algarobeiras, em propriedades localizadas no semi-árido pernambucano. Os proprietários relataram a ocorrência de casos esporádicos com sinais característicos e perda de peso crônica (Fig.1 e 2) devido à lenta evolução da doença. Clinicamente foram observados atrofia da musculatura da face com maior intensidade do masseter, mandíbula pendulosa, protrusão de língua (Fig.3), dificuldade de apreensão e mastigação dos alimentos, desvio lateral da cabeça durante a mastigação ou ruminação, salivação excessiva, disfagia e hi-

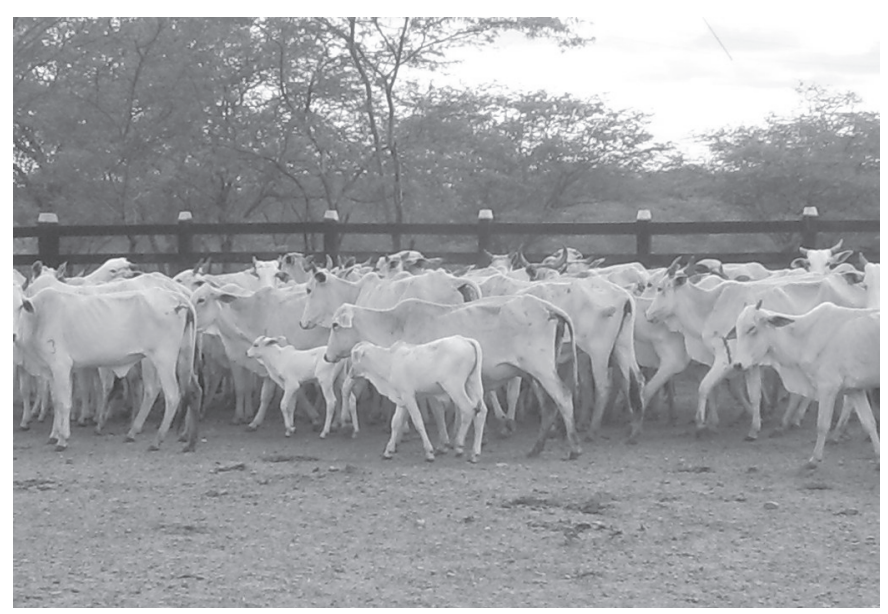

Fig.1. Aspecto das fêmeas adultas da raça Nelore no surto de intoxicação por Prosopis juliflora na propriedade localizada no município de Custódia, Pernambuco.

Quadro 1. Dados epidemiológicos de 3 surtos de intoxicação espontânea por algaroba (Prosopis juliflora) em bovinos no Estado de Pernambuco

\begin{tabular}{|c|c|c|c|c|c|c|c|c|c|c|}
\hline \multirow[t]{2}{*}{ Surto } & \multirow[t]{2}{*}{ Bovino } & \multirow[t]{2}{*}{ Idade } & \multirow[t]{2}{*}{ Sexo $^{a}$} & \multirow[t]{2}{*}{ Raça } & \multirow{2}{*}{$\begin{array}{c}\text { Sistema } \\
\text { de criação }\end{array}$} & \multicolumn{3}{|c|}{ Número de bovinos } & \multirow{2}{*}{$\begin{array}{l}\text { Mês/ano da } \\
\text { ocorrênciab }^{\text {b }}\end{array}$} & \multirow[t]{2}{*}{ Município } \\
\hline & & & & & & Sob risco & Doentes & Mortos & & \\
\hline \multirow[t]{3}{*}{1} & 1 & 2,5 anos & $\mathrm{F}$ & $\mathrm{Hol}^{\mathrm{C}}$ x Gir & Extensivo & 58 & $1^{d}$ & 0 & Outubro 2006 & lati \\
\hline & 2 & $>10$ anos & $\mathrm{F}$ & Nelore & Extensivo & 1206 & $52^{d}$ & $112^{e}$ & Junho 2007 & Custódia \\
\hline & 3 & $>10$ anos & $\mathrm{F}$ & Nelore & Extensivo & & & & & \\
\hline \multirow[t]{2}{*}{2} & 4 & $>10$ anos & $\mathrm{F}$ & Nelore & Extensivo & & & & & \\
\hline & 5 & $>10$ anos & $\mathrm{F}$ & Nelore & Extensivo & & & & & \\
\hline 3 & 6 & 6 anos & M & $\mathrm{Hol}^{\mathrm{C}}$ x Gir & Extensivo & 38 & $1^{d}$ & 0 & Junho 2007 & Buíque \\
\hline \multicolumn{11}{|c|}{$\begin{array}{l}\text { a } F=\text { fêmea, } M=\text { macho. } \\
\text { b Mês de realização do exame clínico na CBG, UFRPE. } \\
\text { c Holandesa. } \\
\text { d Número de animais acometidos pela intoxicação na data do exame clínico. } \\
\text { e Número total de animais mortos }(n=84) \text { e abatidos }(n=28) \text { no decorrer de } 8 \text { meses subseqüentes à visita clínica à } \\
\text { propriedade. }\end{array}$} \\
\hline
\end{tabular}

Pesq. Vet. Bras. 29(3):233-240, março 2009 


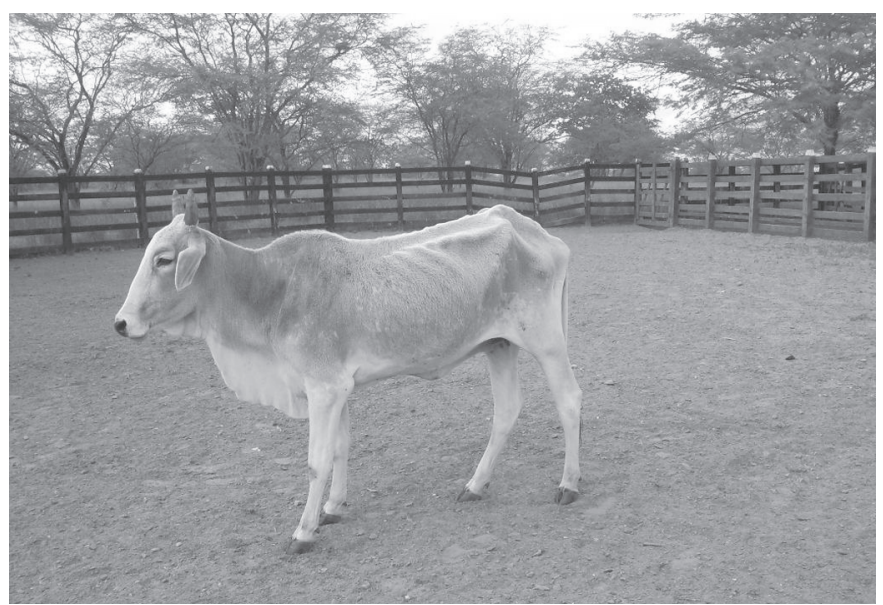

Fig.2. Aspecto do Bovino 3 apresentando escore corporal ruim. Ao fundo, presença de diversas algarobeiras.
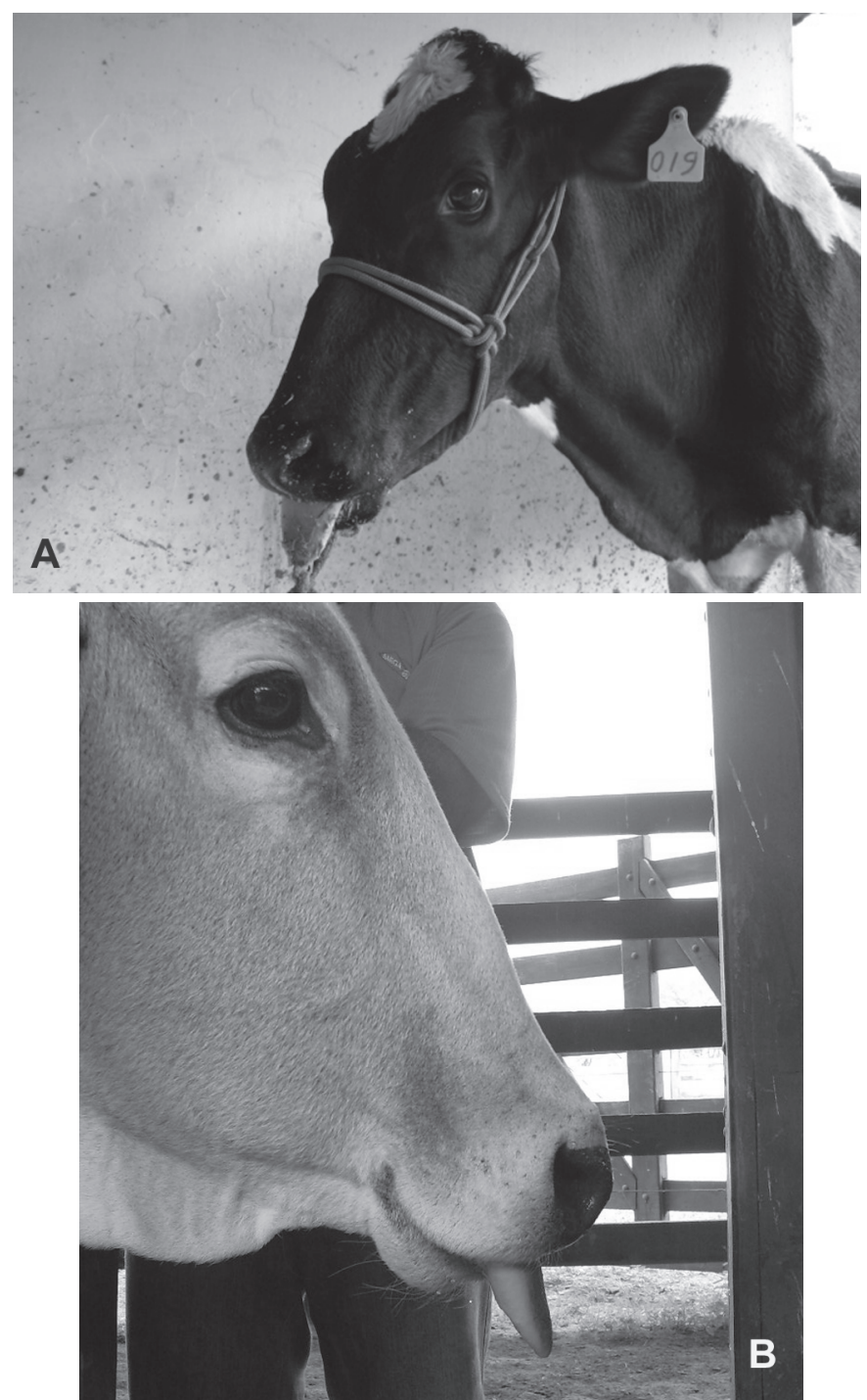

Fig.3. (A) Bovino 1 e (B) Bovino 3 apresentando protrusão de língua devido atrofia dos masseteres em conseqüência de lesões no núcleo motor do $\mathrm{V}$ par de nervo craniano (trigêmeo).
Quadro 2. Achados clínicos em seis casos de intoxicação espontânea por algaroba (Prosopis juliflora) em bovinos no Estado de Pernambuco

\begin{tabular}{|c|c|c|}
\hline Característica & Achado clínico & $\begin{array}{c}\text { Número de } \\
\text { animais }\end{array}$ \\
\hline \multirow[t]{2}{*}{ Comportamento } & Ativo & 3 \\
\hline & Calmo & 3 \\
\hline \multirow[t]{3}{*}{ Atitude } & Decúbito esterno lateral & \\
\hline & (não assumindo estação) & 2 \\
\hline & Estação & 4 \\
\hline \multirow[t]{3}{*}{ Grau de desidratação } & Grave & 1 \\
\hline & Moderado & 4 \\
\hline & Leve & 1 \\
\hline \multirow[t]{2}{*}{ Escore corporal } & II & 4 \\
\hline & I & 2 \\
\hline \multirow[t]{2}{*}{ Mucosas } & Rosadas & 3 \\
\hline & Rosa-Pálidas & 3 \\
\hline \multirow[t]{2}{*}{ Salivação excessiva } & Presente & 2 \\
\hline & Ausente & 4 \\
\hline \multirow[t]{2}{*}{ Atrofia dos masseteres } & Presente & 6 \\
\hline & Ausente & 0 \\
\hline \multirow[t]{2}{*}{ Tônus de língua } & Presente & 4 \\
\hline & Diminuído & 2 \\
\hline \multirow{2}{*}{ Protrusão de língua } & Presente & 5 \\
\hline & Ausente & 1 \\
\hline \multirow{2}{*}{$\begin{array}{l}\text { Desvio lateral da cabeça } \\
\text { durante mastigação }\end{array}$} & Presente & 6 \\
\hline & Ausente & 0 \\
\hline \multirow{2}{*}{$\begin{array}{l}\text { Mandíbula pendulosa à } \\
\text { movimentação manual }\end{array}$} & Presente & 6 \\
\hline & Ausente & 0 \\
\hline \multirow[t]{2}{*}{ Sensibilidade da face } & Presente & 6 \\
\hline & Ausente & 0 \\
\hline \multirow[t]{2}{*}{ Disfagia } & Presente & 6 \\
\hline & Ausente & 0 \\
\hline \multirow[t]{3}{*}{ Temperatura retal } & Normal $\left(38,0-39,0^{\circ} \mathrm{C}\right)$ & 4 \\
\hline & Baixa $\left(<38,0^{\circ} \mathrm{C}\right)$ & 1 \\
\hline & Muito Baixa $\left(<36,0^{\circ} \mathrm{C}\right)$ & 1 \\
\hline $\begin{array}{l}\text { Freqüência cardíaca } \\
\text { (batimentos/minuto) }\end{array}$ & Normal (60-80) & 6 \\
\hline \multirow{2}{*}{$\begin{array}{l}\text { Freqüência respiratória } \\
\text { (movimentos/minuto) }\end{array}$} & Normal (24-36) & 4 \\
\hline & Baixa $(\leq 20)$ & 2 \\
\hline \multirow[t]{2}{*}{ Motilidade ruminal } & Normal & 4 \\
\hline & Reduzida & 2 \\
\hline \multirow[t]{2}{*}{ Plenitude ruminal } & Moderadamente cheio & 1 \\
\hline & Moderadamente vazio & 5 \\
\hline \multirow[t]{2}{*}{ Motilidade intestinal } & Fisiológica & 4 \\
\hline & Reduzida & 2 \\
\hline \multirow[t]{2}{*}{ Fezes } & Ressecadas & 4 \\
\hline & Fisiológicas & 2 \\
\hline
\end{tabular}

potonia lingual em alguns animais. A freqüência dos sinais observados em seis bovinos afetados apresentamse no Quadro 2.

À data de atendimento, os Bovinos 1 e 6 eram casos únicos nas respectivas propriedades e apresentavam histórico de perda de peso progressiva há 4 e 7 meses, respectivamente. No Bovino 1, os sinais iniciaram após o parto. Os proprietários relataram o início da sintomatologia em junho de 2006 e dezembro de 2007, além da ingestão de grande quantidade de vagens de algaroba por ambos os animais na safra do ano anterior. Os Bovinos 2, 3, 4 e 5 compunham parte de numeroso rebanho em que vários animais apresentavam a mesma sintomatologia. Nesta propriedade, no município de Custódia-PE, ocorreram casos esporádicos desde o ano de 2002, sen- 
do realizada a mudança dos animais afetados para locais com melhor oferta de forragem e o posterior comércio para abate daqueles que não se recuperavam. Em junho de 2007 foi realizada visita à propriedade; a doença foi observada em $52(4,31 \%)$ animais de um total de 1206 bovinos ( 845 bovinos da raça Nelore e 361 mestiços). O aparecimento dos sinais clínicos tinha ocorrido nos meses de novembro e dezembro do ano de 2006, apresentando assim evolução clínica de aproximadamente 8 meses. Foram acometidas apenas fêmeas adultas em lactação com idade de 4 a 12 anos, apesar da presença de reprodutores e bezerros no mesmo lote.

Após o diagnóstico clínico durante a visita à propriedade, orientou-se transferência dos animais acometidos para local onde não existisse a planta e melhora da alimentação, assim como administração de complexos vitamínicos com o intuito de promover ganho de peso para futuro abate. Para evitar a ocorrência de futuros casos preconizou-se a adoção de algumas medidas, dentre elas a redução da quantidade de árvores aumentando o espaçamento entre as algarobeiras e evitar o acesso direto dos animais às vagens. Após um ano, em agosto de 2008, o proprietário mencionou que no decorrer de 8 meses subseqüentes à adoção das medidas (até fevereiro de 2008), mais 60 bovinos foram acometidos pela intoxicação totalizando $112(9,28 \%)$ animais, sendo 96 (85,71\%) bovinos da raça Nelore e $16(14,3 \%)$ bovinos mestiços (Nelore $x$ Holandesa). Os bovinos afetados $(n=52)$ e àqueles que adoeceram após a visita clínica $(n=60)$ foram confinados, observando-se que 28 (25\%) apresentaram ganho de peso após confinamento e foram abatidos e 84 (75\%) morreram em decorrência da caquexia e desnutrição, mesmo confinados. Desde então não houve mais casos. O rebanho caprino consistia de aproximadamente 380 animais e ocorriam casos esporádicos da doença nas mesmas áreas em que permaneciam os bovinos. Desde 2002 tinham sido afetados 50 caprinos. Não houve relatos da ocorrência da doença em ovinos, apesar desta espécie ser a mais numerosa na propriedade com um rebanho total de 2.015 cabeças.

Após dividir o rebanho desta fazenda em dois grupos (bovinos Nelore e mestiços), subdividir cada grupo em doentes e não doentes e inserir os dados no programa estatístico, a razão de chances (odds ratio) foi de 2,76 com intervalo de confiança de 1,603 a 4,763 $(\mathrm{P}<0,05)$.

Os valores hematológicos, da proteína plasmática total e do fibrinogênio plasmático são apresentados no Quadro 3. As alterações da série vermelha observadas foram anemia microcítica normocrômica e anemia normocítica normocrômica, no Bovino 1 e 6, respectivamente. Os leucogramas encontravam-se normais para a espécie (Kramer 2000), com exceção do Bovino 6, que revelou leucocitose por neutrofilia com leve desvio para a esquerda regenerativo, sendo evidenciada a inversão da relação neutrófilos: linfócitos, além de hipoproteinemia e hiperfibrinogenemia. Essas alterações sugestivas de um processo inflamatório agudo ocorreram, provavelmente,
Quadro 3. Valores hematológicos, da proteína plasmática total e do fibrinogênio plasmático em quatro casos de intoxicação espontânea por algaroba (Prosopis juliflora) em bovinos no Estado de Pernambuco

\begin{tabular}{|c|c|c|c|c|c|}
\hline Parâmetros & Bovino 1 & Bovino 3 & Bovino 4 & Bovino 6 & $\begin{array}{l}\text { Valores de } \\
\text { referência }\end{array}$ \\
\hline Hematócrito (\%) & 24 & 29 & 32 & 22 & $24-46$ \\
\hline 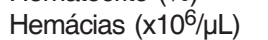 & 6,44 & 5,73 & 7,65 & 4,6 & $5-10$ \\
\hline Hemoglobina (g/dL) & 7,29 & 10,67 & 11,34 & 7,9 & $8-15$ \\
\hline $\operatorname{VCM}(f L)^{a}$ & 37,27 & 50,61 & 41,83 & 47,82 & $40-60$ \\
\hline $\mathrm{CHCM}(\%) \mathrm{b}$ & 30,37 & 35,79 & 35,43 & 35,90 & $30-36$ \\
\hline PPT $(\mathrm{g} / \mathrm{dL})^{\mathrm{c}}$ & 7,6 & 7,3 & 7,1 & 6,6 & $7,0-8,5$ \\
\hline $\mathrm{FP}(\mathrm{mg} / \mathrm{dL}) \mathrm{d}$ & 300 & 300 & 400 & 800 & $300-700$ \\
\hline Leucócitos totais $(/ \mu \mathrm{L})$ & ) 10050 & 7250 & 9850 & 13600 & $4-12$ \\
\hline Neutrófilos $(/ \mu \mathrm{L})$ & 3420 & 3043 & 3743 & 6402 & $600-4000$ \\
\hline Linfócitos (/ $\mu \mathrm{L})$ & 5186 & 4133 & 6107 & 5434 & $2500-7500$ \\
\hline 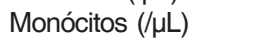 & 642 & - & - & 504 & $25-840$ \\
\hline 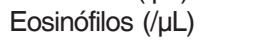 & 802 & - & - & 1260 & $0-2400$ \\
\hline 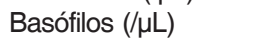 & - & 73 & - & - & $0-200$ \\
\hline
\end{tabular}

a Volume corpuscular médio, ${ }^{b}$ concentração de hemoglobina corpuscular média, ${ }^{\mathrm{c}}$ proteína plasmática total, ${ }^{\mathrm{d}}$ fibrinogênio plasmático, ${ }^{\mathrm{e}} \mathrm{Kramer}$ (2000).

porque o animal tinha sido descornado cirurgicamente, seis dias antes, por um prático. Os Bovinos 3 e 4 apresentaram valores de proteína plasmática total próximos ao limite inferior para bovinos. Os demais valores encontravam-se nos limites fisiológicos para a espécie (Kramer 2000).

Na necropsia do Bovino 2 foram visualizados: diminuição de volume e coloração acinzentada dos músculos masseteres (Fig.4); ausência de panículo adiposo subcutâneo; hipotrofia gelatinosa da gordura visceral, principalmente da pericárdica; presença de algumas vagens de algaroba no rúmen e retículo; hemorragias petequiais subepicárdicas, no sulco paraconal e ventrículo esquerdo, além de sufusões subendocárdicas no ventrículo esquerdo e direito; enfisema pulmonar intersticial; e congestão cerebral. Histologicamente foi observada degeneração neuronal no núcleo motor do nervo trigêmeo. Alguns neurônios apresentavam coloração irregular da substância

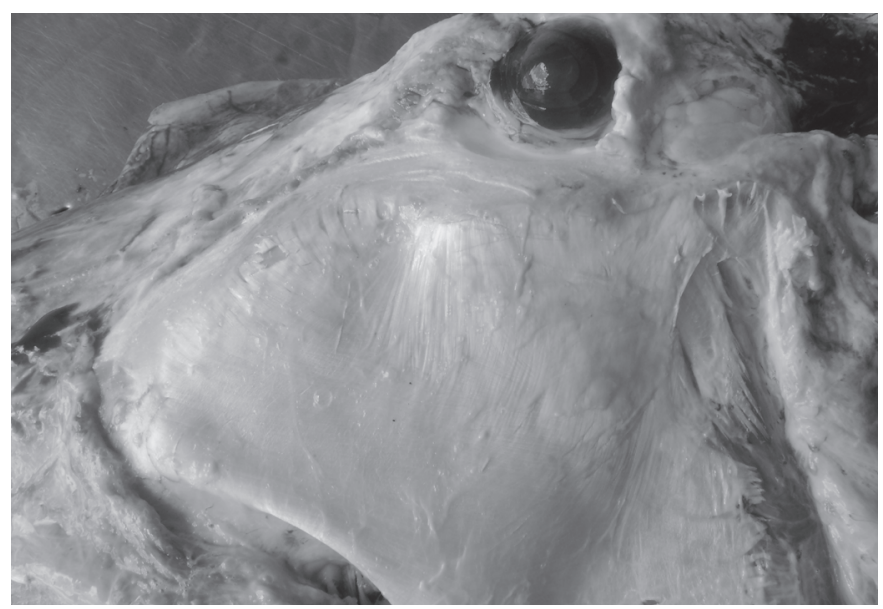

Fig.4. Bovino 2. Fêmea adulta da raça Nelore com ligeira depressão na musculatura do masseter, indicando atrofia. 


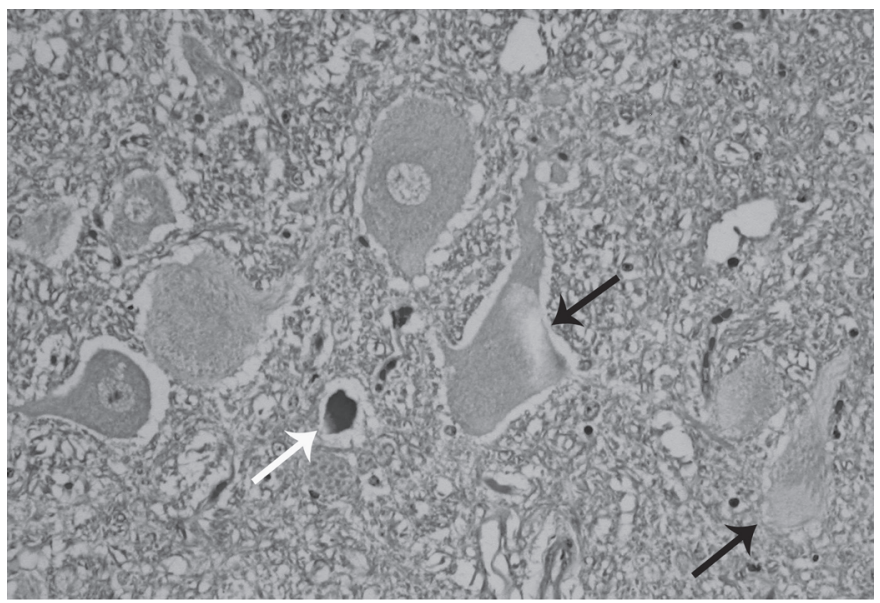

Fig.5. Tronco encefálico. Núcleo motor do nervo trigêmeo. Observam-se neurônios com distribuição irregular da substância de Nissl (setas pretas) e esferóides axonais (seta branca). HE, obj.20x.

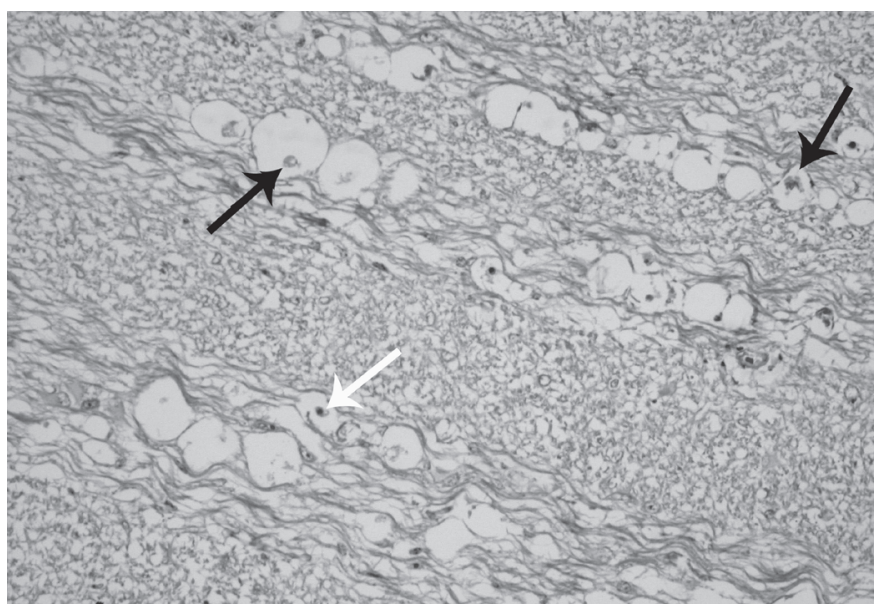

Fig.6. Tronco encefálico. Observam-se as raízes do nervo trigêmeo com degeneração Walleriana, caracterizada por vacúolos em cadeia, ocasionalmente contendo restos de axônios (setas pretas) ou macrófagos (seta branca). HE, obj.20x.

de Nissl (Fig.5), enquanto que outros estavam contraídos com cromatólise e núcleo picnótico. Raros esferóides axonais eram observados (Fig.5). Nas raízes do nervo trigêmeo observou-se degeneração Walleriana, com dilatação da bainha de mielina e presença de vacúolos, geralmente em cadeia, contendo, ocasionalmente, restos axonais ou macrófagos (Fig.6). O músculo masseter apresentava severa atrofia por denervação, com diminuição do tamanho das fibras, presença de numerosas fibras triangulares e desaparecimento de fibras com substituição por tecido conectivo (Fig.7).

\section{DISCUSSÃO}

O diagnóstico de intoxicação por vagens de Prosopís juliflora foi baseado nos sinais clínicos, alterações histológicas características (Tabosa et al. 2000a, 2004, 2006), e histórico de ingestão de vagens durante prolongado

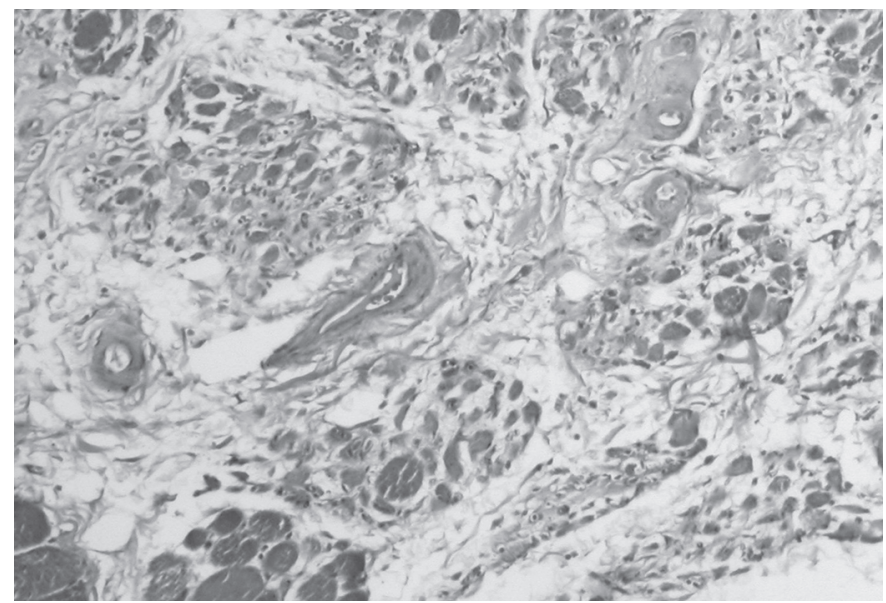

Fig.7. Músculo masseter. Observa-se atrofia de fibras caracterizadas por fibras consideravelmente diminuídas de tamanho, muitas triangulares. No ângulo inferior esquerdo observam-se fibras de tamanho próximo ao normal. Entre os grupos de fibras observa-se proliferação de tecido conectivo. HE, obj.20x.

período de tempo. No estudo epidemiológico constatouse que alguns casos de intoxicação são observados pelos produtores nas épocas em que não há disponibilidade de vagens de algaroba nos pastos. Isto pode ter resultado do fato de que a observação dos sinais por parte dos produtores ocorra após o início da seca, quando diminui a disponibilidade de forragem e os animais apresentam problemas de desnutrição. Outra possibilidade é de que as lesões ocorridas durante a época de produção de vagens de algaroba (outubro-dezembro) sejam progressivas e, em alguns bovinos, os sinais somente iniciem alguns meses após.

Enquanto em duas fazendas a doença teve uma prevalência baixa $(1,72 \%$ e 2,63\%), no surto em Custódia a prevalência foi de 9,28\% (112 animais), comprovando a alta morbidade da intoxicação, que pode acometer até $50 \%$ dos bovinos em algumas fazendas (Dantas \& Menezes 1994). Todos os surtos ocorreram em municípios do Agreste e Sertão Pernambucano onde as vagens de algaroba constituem uma parte importante da alimentação a partir do mês de outubro durante a época seca, quando há maior carência de forragem. Os animais mais afetados foram fêmeas adultas em lactação (Bovinos 1, 2, 3, 4 e 5). Tal fato pode ser devido a maior ingestão de vagens decorrentes de maiores necessidades de proteínas e energia desses animais ou ao fato de que por permanecerem mais tempo na propriedade, as fêmeas em reprodução ingerem vagens de algaroba por dois ou mais anos consecutivos. A razão de chances de 2,76 encontrada, quando comparada a prevalência em bovinos da raça Nelore com a dos animais mestiços, significa que os primeiros possuem 2,76 vezes mais chance de apresentar a enfermidade quando comparados aos bovinos mestiços nas mesmas condições (Schlesselman 1982), o que sugere a maior susceptibilidade da raça Nelore; no entanto, como não foi possível estabelecer a prevalência 
por faixa etária, não pode ser descartada a possibilidade de que as fêmeas dessa raça fossem mais afetadas por serem mais velhas.

Os sinais clínicos observados são semelhantes aos descritos anteriormente (Tabosa et al. 2006) e estão associados a disfunções de diversos nervos cranianos incluindo o trigêmeo, hipoglosso, vago e glossofaríngeo, assim como a deficiência nutricional pela dificuldade na apreensão, mastigação e ruminação de alimentos.

A recuperação observada em alguns bovinos após serem confinados ocorreu devido a que esses animais apresentavam os estágios iniciais da intoxicação. No entanto, a maioria dos casos apresentava sinais irreversíveis e acabaram morrendo, mesmo após o confinamento. Ainda foram relatados casos esporádicos em caprinos e nenhum em ovinos, achados semelhantes aos de Lima et al. (2004) na Paraíba, que reportam a susceptibilidade da espécie caprina à intoxicação, assim como a resistência de ovinos, que podem ingerir rações contendo $70-100 \%$ de vagens de algaroba, durante um ano, sem ser afetados.

Os achados macroscópicos apenas reportam as lesões decorrentes da caquexia oriunda da dificuldade na alimentação e ruminação encontrada em todos os animais, como ausência de panículo adiposo subcutâneo e hipotrofia gelatinosa da gordura pericárdica e visceral. A presença de vagens de algaroba no rúmen e retículo confirma a ingestão da planta por este animal, assim como observado em diversos outros casos, que apresentavam ainda cascas ou as próprias vagens no rúmen mesmo após 3 meses de confinamento em local onde não existia a planta (Tokarnia et al. 2000). Os achados histológicos no núcleo motor do trigêmeo são semelhantes aos mencionados anteriormente, no entanto chama a atenção a ausência da vacuolização do pericário descrita anteriormente em caprinos (Tabosa et al. 2000a, Lima et al. 2004) e bovinos (Tabosa et al. 2006). Esta ausência já foi constatada em outros casos espontâneos da intoxicação em bovinos (Tabosa et al. 2004) e sugere que para a melhor visualização desta lesão nesta espécie são necessários cortes semifinos, como realizado por Tabosa et al. (2006). Isto faz com que a lesão mais facilmente evidenciada seja a degeneração Walleriana das raízes dos nervos cranianos, principalmente o trigêmeo. Estas lesões associadas às lesões neuronais sugerem que há uma perda progressiva de neurônios, que é, provavelmente, responsável pela irreversibilidade da doença. A perda da função neuronal, por degeneração e/ou desaparecimento, causa atrofia neurogênica bilateral, responsável pelos sinais crônicos irreversíveis. É provável que a recuperação observada em casos iniciais da doença deva-se a reversão da degeneração neuronal (dilatação mitocondrial) antes da morte das mesmas e à estabilização e recuperação da atrofia muscular, antes de ocorrer perda de fibras e substituição por tecido gorduroso ou fibroso.

Este trabalho comprova que a intoxicação por algaroba é uma doença importante em bovinos no Estado de Pernambuco, assim como em outras regiões do Nordes- te. Para evitar a intoxicação, recomenda-se que a utilização de vagens ou farelo de algaroba na dieta não ultrapasse o percentual de $30 \%$, por no máximo seis meses (Riet-Correa \& Méndez 2007). No caso de animais a campo, em áreas com algaroba, sugere-se que os animais não permaneçam nas mesmas por períodos maiores que 60 dias (Riet-Correa et al. 2003). Para complementar essas medidas de controle é necessário comprovar a maior susceptibilidade de animais de diferentes raças à intoxicação, como sugerido com a raça Nelore neste trabalho, e determinar se as lesões são progressivas ou se somam de um ano para outro fazendo com que animais adultos que consomem vagens por dois ou mais anos seguidos sejam mais afetados.

$P$. juliflora é uma espécie invasora de cultivos e áreas úmidas, de baixio, formando aglomerados e excluindo outras espécies por sombreamento, impedindo o desenvolvimento da mata ciliar. Exporta, por transpiração, entre 30 e 75 litros de água por dia constituindo sério problema para os recursos hídricos (Instituto Hórus de Desenvolvimento e Conservação Ambiental 2008). No entanto, considerando o alto custo para a sua erradicação, há possibilidades de utilizar a algaroba como fonte de forragem para ajudar na solução das graves crises forrageiras que ocorrem durante a estação seca no semi-árido. Para isso devem ser considerados alguns fatores: evitar a difusão da planta e melhorar a sua produção mediante raleio ou outras técnicas de manejo; utilizar um manejo alimentar correto para evitar intoxicações, considerando a diferente susceptibilidade de bovinos, caprinos e ovinos e o tempo necessário para ocorrer à intoxicação; desenvolver outras técnicas de utilização da algaroba incluído a produção de lenha, carvão, estacas e alimentos para humanos, que poderiam tornar a sua exploração mais econômica. É importante, no entanto, o desenvolvimento de pesquisas, nas áreas invadidas por algaroba, para a sua provável utilização na geração de renda pelas comunidades afetadas incluindo seus diferentes usos, a utilização de variedades adequadas para esses usos e o manejo sustentável da planta. Esses dados são necessários para determinar os custos, a rentabilidade e a sustentabilidade da utilização da algaroba nas áreas invadidas pela planta.

Agradecimentos.- Projeto financiado parcialmente pelo MCT/CNPq, Programa Institutos do Milênio (Proc.420012/2005-2). Agradece-se ao Prof. Dr. Ivon Macedo Tabosa pela revisão do manuscrito.

\section{REFERÊNCIAS}

Azevedo G. 1961. Algaroba. $2^{\underline{a}}$ ed. Serviço de Informação Agrícola, Rio de Janeiro. 32p.

Bacca S.F., Vallenas A., Novoa C., Ochoa J. \& Cueva S. 1963/66. Estudio experimental de la "coquera" em caprinos. Revta. Fac. Med. Vet., Lima, 18/20:131-159.

Dantas J.R.F. \& Menezes R.V.1994. UFPB, UFBA e USP estudam "cara torta", doença que acomete bovinos na Paraíba, Pernambuco e Rio Grande do Norte. Boletim Informativo do CRMV-PB, jan/fev.

Dirksen G., Gründer H.D. \& Stöber M. 1993. Rosenberger, Exame Clínico dos Bovinos. $3^{a}$ ed. Guanabara Koogan, Rio de Janeiro. 419p. 
Dollahite J.W. 1964. Management of the disease produced in cattle on an unbalanced diet of mesquite beans. South. Vet. 17(4):93-295.

Figueiredo L.J.C., Távora J.P.F., Ferreira M.M., Simões S.V.D. \& Dantas J. 1996. Estudo clínico e anátomo-patológico da doença "cara torta" em bovinos no Nordeste Brasileiro. Arq. Esc. Med. Vet. UFBA, Salvador, 18(1):175-183.

Instituto Hórus de Desenvolvimento e Conservação Ambiental 2008. http://www.institutohorus.org.br/download/fichas/Prosopis_juliflora.htm. Acessado em 30 de outubro de 2008.

Jain N.C. 1993. Essentials of Veterinary Hematology. Lea and Febinger, Philadelphia. 417p.

Kingsbury J.M. 1964. Poisonous Plants of the United States and Canada. Prentice-Hall, Englewood Cliffs, New Jersey, p.349-351.

Kramer J.W. 2000. Normal hematology of cattle, sheep and goats, p.1075-1084. In: Feldman B.F., Zinkl J.G. \& Jain N.C. (Ed.), Schalm's Veterinary Hematology. 5th ed. Williams and Wilkins, Philadelphia.

Lima E., Riet-Correa F., Amorin S.L. \& Sucupira Júnior G. 2004. Intoxicação por favas de Prosopis juliflora (algaroba) em caprinos no Nordeste do Brasil. Pesq. Vet. Bras. 24(Supl.):36-37.

Mahgoub O., Kadim I.T., Johnson E.H., Srikandakumar A., AI-Saqri N.M., Al-Abri A.S. \& Ritchie A. 2005a. The use of a concentrate containing Meskit (Prosopis juliflora) pods and date palm byproducts to replace commercial concentrate in diets of Omani sheep. Anim. Feed Sci. Technol. 120:33-41.

Mahgoub O., Kadim I.S., Forsberg N.E., Al-Ajmi D.S., Al-Saqry N.M., Al-Abri A.S. \& Annamalai K. 2005b. Evaluation of Meskit (Prosopis juliflora) pods as a feed for goats Anim. Feed Sci. Technol. 121:319327.

Mayhew I.G. 1989. Large Animal Neurology: A handbook for veterinary clinicians. Lea and Febiger, Philadelphia. 380p.

Ravikala K., Patel A.M., Murthy K.S. \& Wadhwani K.N. 1995. Growth efficiency in feedlot lambs on Prosopis juliflora based diets. Small Rumin. Res. 16:227-231.

Riet-Correa F., Riet-Correa G. \& Schild A.L. 2002. Importância de exame clínico para o diagnóstico das enfermidades do sistema nervoso em ruminantes e eqüídeos. Pesq. Vet. Bras. 22(4):161-168.

Riet-Correa F., Tabosa I.M., Azevedo E.O., Medeiros R.M., Simões S.V.D., Dantas A.F., Alves C.J., Nobre V.M.T., Athayde A.C., Gomes A.A. \& Lima E.F. 2003. Doença dos ruminantes e eqüinos no semiárido da Paraíba. Semi-árido em Foco 1:60-62.

Riet-Correa F. \& Méndez M.C. 2007. Intoxicações por plantas e micotoxinas, p.99-222. In: Riet-Correa F., Schild A.L., Lemos R.A.A. \& Borges
J.R.J. (Ed.), Doenças de Ruminantes e Eqüídeos. Vol.2. Gráfica e Editora Palotti, Santa Maria.

Schlesselman J.D. 1982. Case Control Studies. Oxford University Press, London. 354p.

Silva J.H.V., Oliveira J.N.C., Silva E.L., Jordão Filho J. \& Ribeiro M.L.G. 2002a. Uso da farinha integral de vagem de algaroba (Prosopis juliflora (Sw) D.C.) na alimentação de codornas japonesas. Revta Bras. Zootec. 31(3):1789-1795.

Silva J.H.V., Silva E.L., Jordão Filho J., Toledo R.S., Albino L.F.T., Ribeiro M.L.G. \& Couto H.P. 2002b. Valores energéticos e efeitos da inclusão de farinha integral de vagem de algaroba (Prosopis juliflora (Sw.) D.C.) em rações de poedeiras comerciais. Revta Bras. Zoot. 31(6):2255-2264.

Silva D.M., Riet-Correa F., Medeiros R.M.T \& Oliveira O.D. 2006. Plantas tóxicas para ruminantes e eqüídeos no Seridó Ocidental e Oriental do Rio Grande do Norte. Pesq. Vet. Bras. 26(4):223-236.

Stein R.B.S., Toledo L.R.A, Almeida F.Q., Arnaut A.C., Patitucci L.T., Soares Neto J. \& Costa V.T.M. 2005. Uso do farelo de vagem de algaroba (Prosopis juliflora (Swartz) D.C.) em dietas para eqüinos. Revta Bras. Zootec. 34(4):1240-1247.

Tabosa I.M., Souza J.C.A., Barbosa-Filho J.M., Almeida R.N., Graça D.L. \& Riet-Correa F. 2000a. Neuronal vacuolation of the trigeminal nuclei in goats caused by the ingestion of Prosopis juliflora pods (mesquite beans). Vet. Human. Toxicol. 42:155-158.

Tabosa I.M., Pamplona F.V. \& Quintans Júnior A. 2000b. Isolamento e identificação de juliprosopina, um dos constituintes tóxicos da Prosopis juliflora. Revta Bras. Farmacol. 9:11-22.

Tabosa I.M., Riet-Correa F., Simões S.V.D., Medeiros R.M.T. \& Nobre V.M.T. 2004. Intoxication by Prosopis juliflora pods (mesquite beans) in cattle and goats in Northeastern Brazil, p.341-346. In: Acamovic T., Stewart C.S. \& Pannycott T.W. (Ed.), Toxic Plants and other Natural Toxicants. CAB International Publishing, Wallingford, Oxon, UK.

Tabosa I.M., Riet-Correa F., Barros S.S., Summers B.A. Simões S.V.D., Medeiros R.M.T. \& Nobre V.M.T. 2006. Neurohistologic and ultrastructural lesions in cattle experimentally intoxicated with the plant Prosopis juliflora. Vet. Pathol. 43:695-701.

Tokarnia C.H., Döbereiner J. \& Peixoto P.V. 2000. Plantas Tóxicas do Brasil. Editora Helianthus, Rio de Janeiro, p.120-142.

Washburn K.E., Breshears M.A., Ritchey J.W., Morgan S.E. \& Streeter R.N. 2002. Honey mesquite toxicosis in a goat. J. Am. Vet. Med. Assoc. 220:1837-1839.

Winepiscope version 2.0. 2000. College of Medicine and Veterinary Medicine, University of Edinburgh, UK. http://www.clive.ed.ac.uk/ winepiscope/0. Acesso em 23 de outubro de 2008. 